\title{
Distancia promedio de la línea de implantación pilosa frontal y sus estructuras en una población de mujeres colombianas
}

\author{
The Average Distance of the Frontal Hairline and its Structures in a \\ Population of Colombian Women
}

\section{Distância média da linha pilosa frontal no enxerto e suas estruturas numa população de mulheres colombianas}

\author{
Daniela Lynett-Barrera, MD. * \\ Hernando Mosquera-Sanchez, MD., Esp. ** \\ Jessica Inés Vergara, MD., Esp. *** \\ Sergio Eduardo Serrano-Gómez, MD., MSc. ****
}

\begin{abstract}
Resumen
Introducción: La distancia promedio de las medidas de la línea de implantación pilosa frontal ha sido estudiada en múltiples países, encontrándose variaciones importantes entre ellos. A nivel local no existen estudios que nos permitan conocer el valor para la población colombiana. Objetivo: Determinar la distancia promedio de las estructuras de la línea de implantación pilosa frontal en mujeres. Metodología: Estudio transversal, analítico realizado en mujeres sanas mayores de 18 años, valoradas en consulta externa de dermatología y cirugía plástica de la clínica FOSCAL en Bucaramanga, Colombia, que no presentaran patologías capilares o alteraciones cráneo-faciales. A las participantes se les midió las estructuras de la línea de implantación frontal y se recolectaron variables sociodemográficas y dermatológicas. Resultados: Edad media encontrada fue de 34.3 años con rangos entre 18 y 84 años, el fototipo de piel más frecuente fue el III (42.3\%), la frecuencia de pico de viuda fue del $47.6 \%$ con un alto de $9.7 \mathrm{~mm}$ (IC 9.1-10.3) y ancho de 14.2 mm (IC 13.5-14.8). Discusión: Los resultados encontrados difieren de manera estadísticamente significativa de los resultados reportados en Estados Unidos
\end{abstract}

\begin{abstract}
y España; esta diferencia puede deberse al origen étnico; así mismo hay que tener en cuenta el diseño metodológico y la población origen de los estudios fue diferente, lo cual podría explicar también en parte las diferencias. Conclusiones: Las medidas de la línea de implantación pilosa en Colombia son distintas a las reportadas en la población española y americana, por lo que se aconseja el uso de medidas locales en procedimientos de reconstrucción quirúrgica, con el fin de lograr resultados más acordes a la población local; además de tener un punto de referencia para sospechar ciertas enfermedades en estadios iniciales que cursan con retroceso de la línea de implantación. Se requieren estudios prospectivos con mayor tamaño de muestra que nos permitan estudiar variaciones de la línea de implantación pilosa dentro de la misma población, de acuerdo a sus medidas antropométricas y dermatológicas. [Lynett-Barrera $D$, Mosquera-Sanchez H, Vergara JI, Gómez-Serrano SE. Distancia promedio de la línea de implantación pilosa frontal y sus estructuras en una población de mujeres colombianas. MedUNAB 2017; 20(2): 115-122]
\end{abstract}

Palabras clave: Folículo Piloso; Enfermedades del Cabello; Alopecia; Cuero Cabelludo; Estudios Transversales.

* Médica, Residente de especialización en Dermatología, Universidad Autónoma de Bucaramanga, Floridablanca, Santander, Colombia.

** Médico, especialista en Dermatología, director del programa académico de Dermatología de la Universidad Autónoma de Bucaramanga, Fundación Oftalmológica de Santander (FOSCAL), Floridablanca, Santander, Colombia.

*** Médica, especialista en Dermatología, docente del postgrado de Dermatología de la Universidad Autónoma de Bucaramanga, Fundación Oftalmológica de Santander (FOSCAL), Floridablanca, Santander, Colombia.

${ }_{* \star * \star}$ Médico, magíster en Epidemiología, grupo de investigación Epidemiología Clínica, Universidad Autónoma de Bucaramanga, Floridablanca, Santander, Colombia.

Correspondencia: Daniela Lynett Barrera. Circunvalar 29 No. 22-16, Edifico GAIA apartamento 804, Cañaveral, Floridablanca, Santander. Teléfono: + 57 3123564894.E-mail: dlynett@unab.edu.co 


\section{Abstract}

Introduction: The average distance of hairline measurements has been studied in multiple countries, finding important variations among them. At a local level, there are no studies that allow investigators to know the value for the Colombian population. Objective: Determine the average distance of the structures of the frontal hairline in women. Methodology: A cross-sectional analytical study carried out on healthy women over 18 years of age, who were assessed in the outpatient dermatology and plastic surgery departments of the FOSCAL clinic in Bucaramanga and they do not show capillary pathologies or craniofacial alterations. Results: The average age found was 34.3 years with ranging between 18 and 84 years, the most frequent skin phototype was III (42.3\%), the frequency of the widow's peak was $47.6 \%$ with a height of 9.7 $\mathrm{mm}(\mathrm{Cl}$ 9.1-10.3) and width of $14.2 \mathrm{~mm}(\mathrm{Cl}$ 13.5-14.8). Discussion: The results that were found differ in a statistically significant way from the results reported in the United States and Spain; this difference may be due to ethnic origin; likewise, it is necessary to take into account the methodological design and the origin of the population of the studies were different, which could partly explain the differences as well Conclusions: The measurements of the hairline in Colombia are different from those reported in the Spanish and American population; therefore, the use of local measures in surgical reconstruction procedures is recommended in order to achieve more consistent results with the local population. Additionally, this works as a point of reference to suspect about certain diseases in initial stages that occur with backward movement of the implantation line. Therefore, more prospective studies with larger sample sizes are required to allow researchers to study variations of the hairline within the same population, according to their anthropometric and dermatological measurements. [Lynett-Barrera D, Mosquera-Sanchez $H$, Vergara JI, Gómez-Serrano SE. The Average Distance of the Frontal Hairline and its Structures in a Population of Colombian women. MedUNAB 2017; 20(2): 115-122].

Keywords: Hair Follicle; Hair Diseases; Alopecia; Scalp; Cross-Sectional Studies.

\section{Introducción}

La pérdida de cabello femenina es una causa común de consulta en dermatología a nivel mundial, que conduce una morbilidad psicológica importante en las mujeres, debido a la reducción difusa de la densidad del cabello en la cabeza con el retroceso o no de la línea de implantación pilosa frontal que afecta a mujeres de todas las edades(1); debido a estas patologías cada día se hace necesario entender y conocer los patrones normales de implantación pilosa usual en cada una de las poblaciones, ya que las diversas morfologías reportadas en los estudios mundiales difieren considerablemente según la raza y parámetros culturales, todo esto ligado a los patrones de belleza, independientes para cada población $(2,3)$. La incidencia y prevalencia de la alopecia femenina aumenta con la edad (Figura 1) (1), con una incidencia general del $20 \%$ respectivamente $(1,4)$.

\section{Resumo}

Introdução: A distância média das medidas da linha do cabelo frontal foi estudada em vários países, com importantes variações entre elas. No nível local, não há estudos que nos permitam conhecer o valor para a população colombiana. Objetivo: Determinar a distância média das estruturas do cabelo frontal em mulheres. Metodologia: Estudo transversal, analítico realizado em mulheres saudáveis com mais de 18 anos, avaliado em ambulatório de dermatologia e cirurgia plástica na clínica FOSCAL em Bucaramanga, Colômbia, que não apresentou patologias capilares ou alterações craniofaciais. Às participantes foram medidas as estruturas da linha de enxerto frontal e as variáveis sociodemográficas e dermatológicas foram coletadas. Resultados: A idade média foi de 34.3 anos, com intervalos entre 18 e 84 anos, o fotótipo de pele mais frequente foi III $(42.3 \%)$, a frequência de pico da viúva foi de $47.6 \%$ com uma altura de $9.7 \mathrm{~mm}$ (IC 9.1-10.3) e largura $14.2 \mathrm{~mm}$ (IC 13.5-14.8). Discussão: No sentido estatísco, as diferenças são significativas às já reportadas nos Estados Unidos e Espanha. Essa diferença pode ser devido à origem étnica e ao fato de que a metodologia e a origem da população em estudo foi diferente, podendo explicar parte das diferenças. Conclusões: As medidas da linha do cabelo na Colômbia são diferentes das relatadas na população espanhola e americana, por isso é aconselhável usar medidas locais em procedimentos de reconstrução cirúrgica, a fim de alcançar resultados mais consentâneos com a população local; Além de ter um ponto de referência para suspeitar certas doenças nos estágios iniciais que ocorrem com o movimento para trás da linha de implantação. Estudos prospectivos com tamanhos de amostra maiores são necessários para permitirnos estudar variações da linha do cabelo dentro da mesma população, de acordo com suas medidas antropométricas e dermatológicas. [Lynett-Barrera D, Mosquera-Sanchez $H$, Vergara JI, Gómez-Serrano SE. Distância média da linha pilosa frontal no enxerto e suas estruturas numa população de mulheres colombianas. MedUNAB 2017; 20(2): 115-122].

Palavras-chaves: Folículo Piloso; Doenças do Cabelo; Alopecia; Couro Cabeludo; Estudos Transversais.

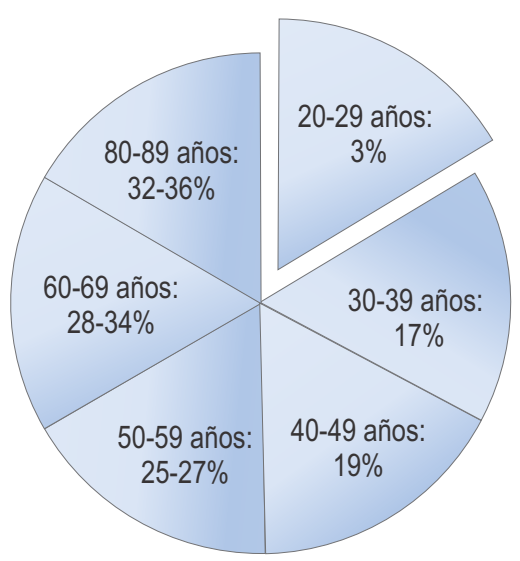

Figura 1. Prevalencia de Alopecia androgenética en la población americana. $(1,4)$. 
En la literatura mundial se han descrito diferentes patrones clínicos y clasificaciones de la alopecia femenina, que guían a la identificación e investigación de los pacientes $(1,5)$; una de las clasificaciones más prácticas es según la presentación temprana o tardía, asociada o no al exceso de andrógenos en mujeres, ya que desde hace varios años se identificaron estos patrones y variantes raciales en varones (4).

A nivel mundial en poblaciones como Estados Unidos, España, y algunos países de Asía se cuenta con estudios de los patrones de implantación pilosa frontal. En 2009 Nusbaum y Fuentefria publicaron las medidas de las líneas de implantación pilosa frontal en 360 mujeres americanas que acudieron a salones de peluquería (6). En 2011 Jung $y$ Cols., describieron 5 formas o patrones de líneas de implantación frontal en 130 mujeres asiáticas elegidas al azar (3); ambos estudios limitados por el criterio de selección utilizado, puesto que no investigaron si las participantes, tanto las que acudieron a salones de peluquería como las elegidas al azar, presentaban algún trastorno dermatológico u hormonal que pudiera influir en las mediciones(2).

En el año 2013, en España, Ceballos realizó un estudio en una población de mujeres españolas que podría compartir algunos rasgos con nuestra población. Este estudio logró determinar el promedio de la línea de implantación pilosa frontal y las demás estructuras que la componen, además, concluyó que los resultados obtenidos en estudios previos no son extrapolables a su población $(2,3,6,7)$.

En Colombia se evidencia un vacío en el conocimiento de la morfología y dimensiones de la línea de implantación pilosa frontal, ya que no se dispone de estudios que aborden el tema; este conocimiento es útil para valorar, graduar y tratar procesos que cursan con retroceso de la línea de implantación como la alopecia frontal fibrosante y la alopecia androgenética femenina de patrón masculino $(2,8,9)$; y también para lograr obtener resultados estéticos más aceptables en los casos candidatos a reconstrucción quirúrgica (10-15). El objetivo de este estudio es determinar la distancia promedio de las estructuras de la línea de implantación pilosa frontal en mujeres.

\section{Metodología}

Estudio transversal, analítico, se seleccionaron trescientas mujeres sanas de todos los grupos etarios, valoradas en consulta externa de dermatología y cirugía plástica de la clínica FOSCAL en Bucaramanga, Colombia. Los criterios de inclusión fueron la edad mayor o igual a 18 años, y aceptar participar en el estudio. Los criterios de exclusión fueron:

1. Mujeres con motivos de consulta o antecedentes de patología hormonal (trastornos menstruales, ciclos anovulatorios, Síndrome de ovario poliquístico).

2. Patología de cuero cabelludo (psoriasis, liquen plano, tiñas) o displasias pilosas.

3. Diagnóstico o sospecha de hiperandrogenismo o SAHA en cualquiera de sus grados (acné, alopecia, hirsutismo, hipertricosis).

4. Sospecha clínica o diagnóstico confirmado de alopecias No cicatriciales o cicatriciales.

5. Estado de embarazo.

6. Antecedente de cirugía facial o de cráneo previa que alteren la anatomía de la línea de implantación pilosa frontal (lifting quirúrgico, cirugía por trauma craneoencefálico, drenaje de hematomas, incisión coronal, etc.).

7. Uso de medicamentos que afecten la línea de implantación pilosa frontal.

8. Pacientes con cambio de género.

Las variables fueron recolectadas por medio de entrevista oral previo consentimiento informado y clasificadas como sociodemográficas: edad, vinculación al sistema de salud, lugar de residencia, estrato social, escolaridad y ocupación; las dermatológicas fueron fototipo de piel, menarquia, menopausia, color de pelo, iris, oleosidad del cuero cabelludo y cara, uso de anticonceptivos orales y tabaquismo. Posteriormente se determinó la presencia de pico de viuda y sus dimensiones, se efectuaron las medidas de los otros componentes de la línea de implantación pilosa frontal tanto para el perfil derecho como izquierdo por medio de un calibrador (Figura 2). Todas las medidas fueron realizadas por los investigadores con el mismo instrumento, previo
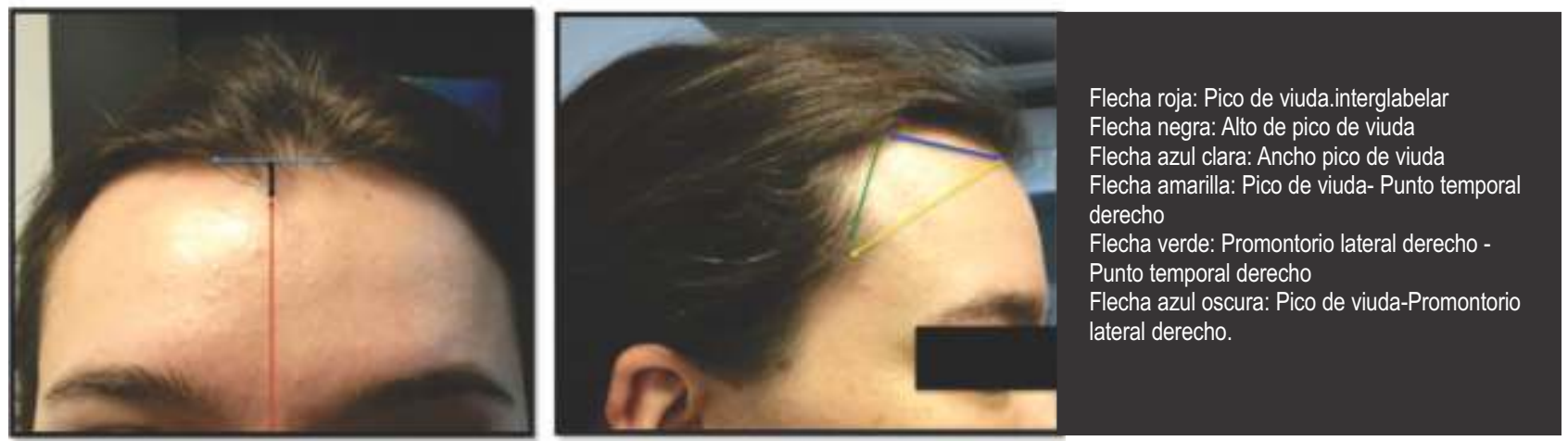

Fuente: Pacientes incluidas en el estudio del servicio de consulta dermatología realizado en la Clínica FOSCAL adaptada de (2), fecha: enero 2017.

Figura 2. Medidas realizadas de la línea de implantación pilosa frontal n=300 
Tabla 1. Edad de los pacientes

\begin{tabular}{lcccr}
\hline Variable & Promedio & IC 95\% & \multicolumn{2}{c}{ Rango ( años) } \\
\hline Edad $^{*}$ & $34.3(14.4)$ & $32.6-35.9$ & 18.0 & 84.0 \\
\hline
\end{tabular}

*Años cumplidos, $n=300$ pacientes.

Tabla 2. Menarquia-Menopausia

\begin{tabular}{lrrrr}
\hline Variable & Promedio & IC 95\% & \multicolumn{2}{c}{ Rango (años) } \\
\hline Edad menarquia* & $12.7(1.5)$ & $12.5-12.9$ & 9.0 & 18.0 \\
Edad menopausia* & $47.1(7.1)$ & $45.2-49.1$ & 30.0 & 57.0 \\
\hline
\end{tabular}

entrenamiento en la Unidad de dermatología de la Universidad Autónoma de Bucaramanga.

Los datos se registraron en un formato de recolección y, posteriormente, se digitaron en una hoja de Excel anonimizada y se exportaron a STATA 14, se realizó análisis univariado y bivariado según las variables y se estimó el percentil 5 y 95 de las medidas para establecer la distribución normal en nuestra población.

Este estudio se presentó de acuerdo con la reglamentación ética vigente (Declaración de Helsinki y Resolución 008430 del 4 de octubre de 1993 del Ministerio de Salud de Colombia) y fue evaluado y aprobado por el comité de ética de la clínica FOSCAL en Bucaramanga, Colombia, clasificado como una investigación sin riesgo.

\section{Resultados}

Trescientas mujeres colombianas fueron incluidas en el análisis, el promedio de edad fue de 34.3 años (DE: 14.4) en un rango de edad comprendido entre los 18 y 84 años (Tabla 1).

Con respecto a la edad de menarquia el promedio encontrado fue de 12.7 años DE (1.5) con rango de edad en años estuvo comprendido entre los 9 y 18 , el $81.3 \%$ de las mujeres en el estudio no presentaban estado de menopausia acorde con el promedio de edad encontrado, el $18.6 \%$ tenían antecedente de menopausia cuyo promedio de edad fue 47 años DE (7.1) con rangos entre los 30 y 57 años (Tabla 2).

Respecto a las características fenotípicas evaluadas se encontró que el fototipo de piel más prevalente en la población fue el III (42.3\%), seguido del IV (39.6\%), las demás características físicas encontradas fueron predominio del color de pelo negro $46 \%$, iris color café oscuro $72 \%$. Respecto a los parámetros relacionados con oleosidad en piel de cara y cuero cabelludo el $52.6 \%$ no refería este signo asociado. El 79.6\% de las mujeres del estudio no consumían anticonceptivos orales y el $95.3 \%$ no tenían consumo actual de tabaco (Tabla 3).
Tabla 3. Variables Dermatológicas

\begin{tabular}{|c|c|c|}
\hline Variable & Porcentaje & IC $95 \%$ \\
\hline \multicolumn{3}{|c|}{ Fototipo de Piel } \\
\hline 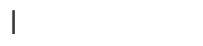 & 0.3 & $0.04-2.3$ \\
\hline$\|$ & 12.6 & $9.3-16.9$ \\
\hline III & 42.3 & $36.8-48.0$ \\
\hline IV & 39.6 & $34.2-45.3$ \\
\hline V & 5.0 & $3.0-8.1$ \\
\hline VI & 0 & 0 \\
\hline \multicolumn{3}{|l|}{ Color Pelo } \\
\hline Negro & 46.0 & $40.4-52.6$ \\
\hline Café oscuro & 39.6 & $34.2-45.3$ \\
\hline Café claro & 9.0 & $6.2-12.8$ \\
\hline Rubio & 4.0 & $2.2-6.9$ \\
\hline Canoso & 1.3 & $0.4-3.5$ \\
\hline \multicolumn{3}{|l|}{ Color Iris } \\
\hline Café oscuro & 72.0 & $66.6-76.8$ \\
\hline Café claro & 2.0 & $15.8-24.9$ \\
\hline Azul & 0.3 & $0.04-2.3$ \\
\hline Verde & 7.6 & $5.1-11.2$ \\
\hline Gris & 0 & 0 \\
\hline \multicolumn{3}{|c|}{ Oleosidad Piel cara } \\
\hline $\mathrm{Si}$ & 47.3 & $41.7-53.0$ \\
\hline No & 52.6 & $46.9-58.2$ \\
\hline \multicolumn{3}{|c|}{ Oleosidad Cuero Cabelludo } \\
\hline $\mathrm{Si}$ & 44.0 & $38.4-49.7$ \\
\hline No & 56.0 & $50.2-61.5$ \\
\hline \multicolumn{3}{|l|}{ Acos } \\
\hline $\mathrm{Si}$ & 20.3 & $16.1-25.2$ \\
\hline No & 79.6 & $74.7-83.8$ \\
\hline \multicolumn{3}{|l|}{ Tabaquismo } \\
\hline $\mathrm{Si}$ & 4.6 & $2.7-7.7$ \\
\hline No & 95.3 & $92.2-97.2$ \\
\hline \multicolumn{3}{|l|}{ Menopausia } \\
\hline Si & 18.6 & $14.6-23.5$ \\
\hline No & 81.3 & $76.4-85.3$ \\
\hline
\end{tabular}

De acuerdo a los objetivos principales del estudio: identifcar la frecuencia de presentación del pico de viuda y sus dimensiones, se encontró que el pico de viuda en las mujeres solo está presente en un $47.6 \%$ de la población, lo cual corresponde a 144 pacientes de la muestra; las dimensiones en milímetros del promedio del ancho del pico de viuda, de estas pacientes, fue $14.2 \mathrm{~mm}(6 \mathrm{a} 29 \mathrm{~mm})$ y el alto $9.7 \mathrm{~mm}$ (3$21 \mathrm{~mm}$ ) respectivamente.

El promedio de medidas de las variables con sus respectivos intervalos de confianza que conforman la línea de implantación pilosa frontal se presentan en la Figura 3 y 4 respectivamente. 


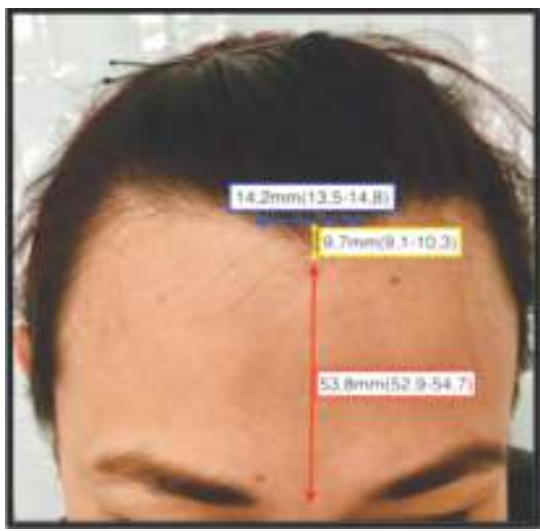

Fuente: Pacientes incluidas en el estudio del servicio de consulta dermatología realizado en la Clínica FOSCAL.

Figura 3. Promedios de la línea de implantación pilosa frontal. Pico de viuda y sus dimensiones $n=300$
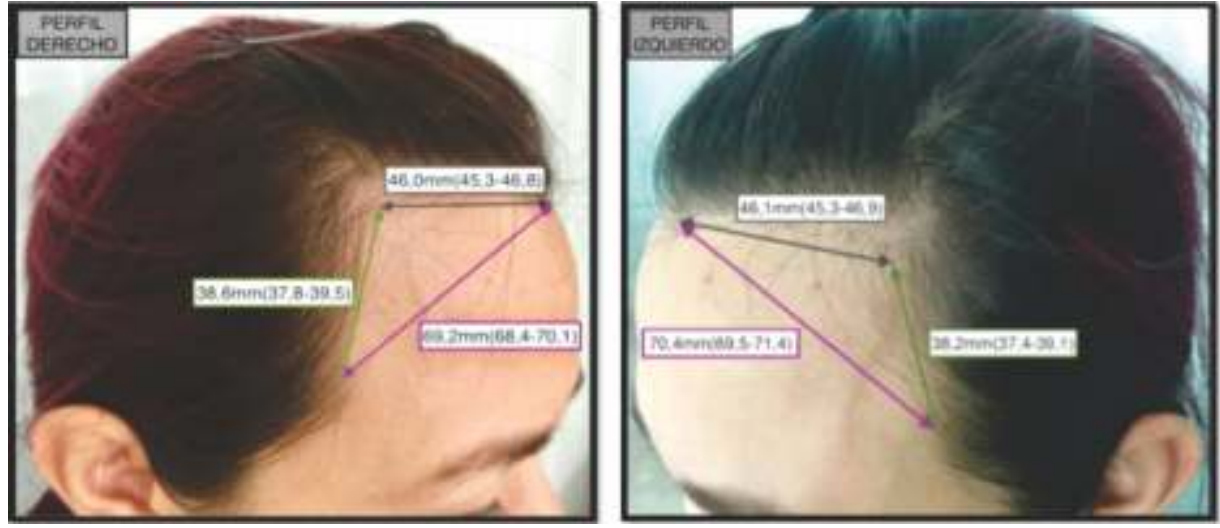

Fuente: Pacientes incluidas en el estudio de consulta dermatología realizado en la Clínica FOSCAL, fecha: marzo 2017,

Figura 4. Promedios de los componentes de la línea de implantación pilosa frontal en ambos perfiles $n=300$

\section{Discusión}

Diversas patologías del cuero cabelludo especialmente el grupo de las alopecias como la alopecia androgenética femenina de patrón masculino, alopecia frontal fibrosante así como anormalidades genéticas o deformidades secundarias de la línea de implantación por intervenciones quirúrgicas, presentan alteraciones en su implantación o experimentan un retroceso progresivo de la línea de implantacion pilosa frontal, trayendo consigo a las pacientes una alta carga emocional con afectación negativa en las múltiples esferas del ser humano, principalmente en la interacción psicosocial; por ello cada día es más frecuente el uso de diversas técnicas tanto quirúrgicas como no quirúrgicas para su reconstrucción, desconociéndose hasta la fecha el promedio de los componentes de la línea de implantacion pilosa frontal en la mujer colombiana $(1,4,16)$.
Con este estudio se ha determinado la frecuencia de presentación del pico de viuda y el promedio de los componentes de la línea de implantación pilosa frontal, en trescientas mujeres de Santander, Colombia, en un periodo de 3 meses, lo que convierte este estudio en la muestra más grande hasta el momento realizada en esta pregunta investigativa en Latinoamérica. Analizando las características sociodemográficas de la población, el promedio de edad fue de 34.3 años con rangos de edad extensos comprendidos entre los 18 y 84 años, comparado con el estudio español publicado en 2013 en 105 mujeres en donde el promedio de edad fue de 29.7 años con rangos entre 18 y 45 años; una de las limitaciones en el estudio español es que evaluó mujeres hasta los 45 años, desconociendo las medidas de la línea de implantación pilosa frontal después de esta edad, en comparación con nuestro estudio en donde evaluó mujeres hasta los 84 años. El límite superior de edad evaluado en la población española (45 años versus 84 años), podría explicar las diferencias en los resultados 
encontrados en las medidas de la línea de implantación frontal en la población colombiana, especialmente en el grupo de menores de 40 años, deconociéndose dichos valores en mujeres mayores de 45 años en España $(2,6)$.

Se encontró que la presencia de pico de viuda en nuestra población es del $47.6 \%$ comparada con un $94.1 \%$ y $81 \%$ reportada en los estudios realizados en España y Estados Unidos respectivamente; estos datos sugieren que la presencia de pico de viuda es una característica frecuente en la población española y americana, pero no en la nuestra, señalando que la presencia del pico de viuda es una característica propia de cada grupo poblacional, sin embargo estos resultados pueden estar influenciados por el tamaño de la muestra. $(2,6)$ (Tabla 4).

Al relacionar la edad distribuida en grupos: menores de 40 años, entre 40-60 años y mayores de 60 años con los componentes de la línea de implantación pilosa frontal se encontraron resultados estadísticamente significativos en las medidas del promontorio lateral a los puntos temporales en el grupo de edad entre los 40 y 60 años $(p=0.005)$ y ( $p=$ $0.0115)$, estos resultado pueden apoyar la hipótesis del retroceso secundario al proceso de envejecimiento, en nuestro estudio encontramos que en las mujeres en algunos componentes de la línea de implantación se asoció con la edad y con la menopausia, la edad promedio del retroceso en nuestro estudio fue a los 47 años, sin embargo a pesar de que los resultados son estadísticamente significativos, presentaron valores limítrofes en el análisis bivariado entre las medidas de la línea de implantación en relación con los grupos etarios y la presencia o ausencia de pico de viuda, por lo que se considera necesario realizar estudios con mayor tamaño muestral que aporten mayor poder estadístico para evaluar estas variables $(2,4,16)$.

El estudio pionero realizado en Estados Unidos por Nusbaum y Fuentefria, publicado en 2009, determinó la frecuencia y los componentes de la linea de implantación pilosa, pero como limitantes de este estudio está la selección de los participantes, la cual fue por conveniencia, sin rigurosos criterios de selección o exclusión, por lo que no se tuvieron en cuenta factores de confunsión asociados que podrían influir en los resultados estadísticos; sin embargo, en el estudio realizado por Ceballos y Priego en una población de mujeres caucásicas de España, publicado en el 2013, tuvieron en cuenta variables que pueden influir en los resultados como la valoración de patologías actuales sistémicas, antecedentes personales, patologías del foliculo piloso, uso de medicamentos, estado de embarazo, raza y otros factores que pueden afectar directamente las medidas de implantación pilosa frontal, una de las fortalezas de nuestro estudio es que estas variables fueron medidas e incluidas para el analísis estadístico $(2,6,17)$.

Se observó que el promedio de la mayoría de las medidas de los componentes de la línea de implantación pilosa frontal de la población española frente a la colombiana son mayores, excepto en la distancia entre el pico de viuda y los promontorios laterales, el cual es menor en la población española por una diferencia de máximo $2 \mathrm{~mm}$; adicionalmente, al comparar dichos resultados con la población americana, se encuentran medidas menores frente a la población española e incluso frente a nuestros resultados, especialmente en las distancias del pico de viuda a los promontorios lateral y de ellos a los puntos temporales; señalando que las medidas de las diversas estructuras que componen la línea de implantación pilosa frontal son mayores en la población española $(2,6,18,19)$ (Tabla 5).

Es de resaltar que en el estudio realizado por Ceballos y Priego, publicado en 2013, se evidencian rangos de cero reportados en las medidas de ancho y alto del pico de viuda lo que significaría que individuos que no presentan pico de viuda, no debían haberse tenido en cuenta de este resultado ya que influye en el analísis final (2,20-22) (Tabla 5).

\section{Conclusiones}

En la literatura analizada no se encontraron estudios realizados en Latinoamérica ni en Colombia, similares al nuestro; es decir, que describan la frecuencia de presentación del pico de viuda o sus dimensiones, así como la distancia promedio de las estructuras que componen la línea de implantación.

Los datos aportados por nuestro estudio permiten tener un acercamiento a las medidas de la población colombiana, y su importancia como punto de referencia para la sospecha, el tamizaje o diagnóstico de ciertas enfermedades en estadios iniciales como la alopecía androgenética femenina de patrón masculino, la alopecia frontal fibrosante $u$ otras

Tabla 4. Comparación Presencia Pico de Viuda Colombia-España-Estados Unidos

\begin{tabular}{lcccccc}
\hline & \multicolumn{2}{c}{ Colombia $n=300$} & \multicolumn{2}{c}{ España $n=103$} & \multicolumn{2}{c}{ EE.UU $n=360$} \\
\hline Pico de Viuda (PV) & Pacientes & Porcentaje & Pacientes & Porcentaje & Pacientes & Porcentaje \\
SI & 144.0 & 47.6 & 97.0 & 94.1 & 293.0 & 81.0 \\
NO & 156.0 & 52.3 & 6.0 & 5.9 & 67.0 & 18.5 \\
\hline
\end{tabular}


Tabla 5. Comparación Variables Línea de implantación Pilosa Frontal a nivel mundial

\begin{tabular}{|c|c|c|c|c|c|c|c|c|c|}
\hline \multirow[b]{3}{*}{ Ancho PV mm* } & \multicolumn{3}{|c|}{ Colombia $n=300$} & \multicolumn{3}{|c|}{ España $n=103$} & \multicolumn{3}{|c|}{ EE.UU $n=360$} \\
\hline & \multirow{2}{*}{$\begin{array}{l}\text { Promedio } \\
14.2(4.1)\end{array}$} & \multicolumn{2}{|c|}{ Rango } & \multirow{2}{*}{$\begin{array}{c}\text { Promedio } \\
21.3\end{array}$} & \multicolumn{2}{|c|}{ Rango } & \multirow{2}{*}{$\begin{array}{c}\text { Promedio } \\
18.3\end{array}$} & \multicolumn{2}{|c|}{ Rango } \\
\hline & & 6.0 & 29.0 & & 0.0 & 50.0 & & 5.0 & 47.0 \\
\hline Alto $\mathrm{PV} \mathrm{mm} \mathrm{mm}^{*}$ & $9.7(3.5)$ & 3.0 & 21.0 & 10.1 & 0.0 & 35.0 & 8.0 & 2.0 & 30.0 \\
\hline PV -Interglabelar mm* & $53.8(8.0)$ & 33.0 & 77.0 & 58.9 & 45.0 & 90.0 & 55.5 & 35.0 & 80.0 \\
\hline $\begin{array}{l}\text { PV -Promontorio lateral } \\
\text { izquierdo* }\end{array}$ & $46.1(7.1)$ & 27.0 & 71.0 & 44.0 & 20.0 & 70.0 & 39.7 & 15.0 & 60.0 \\
\hline $\begin{array}{l}\text { PV-Promontorio lateral } \\
\text { derecho* }\end{array}$ & $46.0(6.8)$ & 31.0 & 64.0 & 44.5 & 25.0 & 75.0 & 37.4 & 10.0 & 57.0 \\
\hline $\begin{array}{l}\text { Promontorio lateral izq- } \\
\text { Punto temporal izq mm }\end{array}$ & $38.2(7.6)$ & 19.0 & 62.0 & 45.4 & 25.0 & 90.0 & 35.1 & 3.0 & 60.0 \\
\hline $\begin{array}{l}\text { Promontorio lateral der- } \\
\text { Punto temporal der } \mathrm{mm}^{*}\end{array}$ & $38.6(7.3)$ & 16.0 & 60.0 & 48.3 & 20.0 & 83.0 & 37.8 & 3.0 & 62.0 \\
\hline PV-punto temporal izq mm & $70.4(8.2)$ & 41.0 & 94.0 & 81.5 & 50.0 & 100.0 & 74.8 & - & - \\
\hline PV-punto temporal der $\mathrm{mm}^{*}$ & $69.2(7.8)$ & 40.0 & 88.0 & 81.1 & 56.0 & 105.0 & 75.1 & - & - \\
\hline
\end{tabular}

(-) No se disponen de datos, promedio y rango en milimetros (mm), PV: pico de viuda, izq: izquierdo, der: derecha.

enfermedades que cursan con retroceso de la linea de implantación correlacionados con la clínica.

Además, nuestros hallazgos pueden ser de gran utilidad en el campo de la cirugía dermatológica, tricología y cirugía plástica en Colombia, ya que aportan referencias propias para las intervenciones quirúrgicas de reconstrucción de la línea de implantación por medio del trasplante capilar.

Se observó que las medidas de las diversas estructuras que componen la línea de implantación pilosa frontal encontradas en nuestra población son menores que las reportadas en España y estados Unidos; estos resultados diferentes para cada una de las tres poplaciones sugieren que estas medidas son caracteríscas específicas de cada población y no se pueden extrapolar a la población general debido a la influencia de los rasgos fenotípicos y raciales de cada población.

Estos resultados muestran la necesidad de la realización de estudios con mayor tamaño muestral y poder estadístico para confirmar algunas de las observaciones estadísticamente significativas encontradas respecto al retroceso de la línea de implantación relacionado con la edad y menopausia especialmente en mujeres mayores de 60 años. Finalmente, se resalta la importancia de la realización de estudios en nuestra población por las razones previamente expuestas.

\section{Responsabilidades éticas}

Protección de personas y animales. Los autores declaran que en esta investigación no se han realizado experimentos en seres humanos ni en animales.

Confidencialidad de los datos. Los autores declaran que han seguido los protocolos de su centro de trabajo sobre la publicación de datos de pacientes.

Derecho a la privacidad y consentimiento informado. Los autores han obtenido el consentimiento de los pacientes referidos. Este documento obra en poder del autor de correspondencia.

\section{Conflicto de intereses}

Los autores declaran no tener conflicto de interés.

\section{Referencias}

1. Singal A, Sonthalia S, Verma P. Female pattern hair loss. Indian J Dermatology, Venereol Leprol [Internet]. 2013;79(5):626. Disponible en: http://www. ncbi.nlm.nih.gov/pubmed/23974580 
2. Ceballos C, Priego C, Méndez C, Hoffner M V., GarcíaHernández MJ, Camacho FM. Estudio de patrones de implantación pilosa frontal en la mujer española caucásica. Actas Dermosifiliogr. 2013; 104(4):311-5.

3. Jung JH, Rah DK, Yun IS. Classification of the female hairline and refined hairline correction techniques for Asian women. Dermatologic Surg. 2011; 37(4):495-500.

4. Tosti a., Piraccini BM, Sisti a., Duque-Estrada B. Hair loss in women. Minerva Ginecol [Internet]. Elsevier Inc.; $2009 ; 61(5): 445-52$. Disponible en: http://dx.doi.org/10.1016/j.sder.2009.01.001.

5. Lee WS, Ro BI, Hong SP, Bak H, Sim WY, Kim DW, et al A new classification of pattern hair loss that is universal for men and women: Basic and specific (BASP) classification. J Am Acad Dermatol. 2007; 57(1):37-46.

6. Nusbaum BP, Fuentefria S. Naturally occurring female hairline patterns. Dermatologic Surg. 2009; 35(6): 907-13.

7. Naz G., Hameed F., Bibi Z., Khatoon D., Sajjad N. Hairline shapes: study of a morphogenetic inheritance trait. Par. J. Biochem. Mol. Biolo. 2014; 47 (1-2):118-20.

8. Atanaskova Mesinkovska N, Bergfeld WF. Hair: What is New in Diagnosis and Management?. Female Pattern Hair Loss Update: Diagnosis and Treatment. Dermato Clin. 2013; 31(1):119-27.

9. Jimenez F. Hair transplantation and female hairlines. Dermatologic Surg. 2011; 37(4):501-2.

10. Park JH. Side-hairline Correction in Korean Female Patients. Plast Reconstr Surg - Glob Open [Internet]. $2015 ; 3(3)$ : e 336 . D i s p o n ible e n: http://content.wkhealth.com/linkback/openurl?sid=WKP TLP:landingpage\&an=01720096-201503000-00006

11. Beehner M. Hairline design in hair replacement surgery. Facial Plast Surg. 2008; 24(4):389-403.

12. Ramirez AL, Ende $\mathrm{KH}$, Kabaker SS. Correction of the high female hairline. Arch Facial Plast Surg. 2011; 11(2):84-90.

13. Kabaker SS, Champagne JP. Hairline Lowering. Facial Plast Surg Clin North Am [Internet]. Elsevier Inc; 2013;
$21(3): 479-86$. D i s p o n i b le e n : http://dx.doi.org/10.1016/j.fsc.2013.05.007

14. Park I., Bang CY,, Kang MJ., Jung JH., Byun JW., Shin J., et al. Female Hairline Preference among Various Segments of the Korean Population. Ann Dermatol. 2014: 26(5):647-9.

15. Park JH. Novel Principles and Techniques to Create a Natural Design in Female Hairline Correction Surgery. Plast Reconstr Surg - Glob Open [Internet]. 2015;

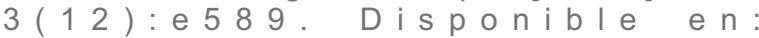
http://content.wkhealth.com/linkback/openurl?sid=WKP TLP:landingpage\&an=01720096-201512000-00003

16. Rassman WR, Pak JP, Kim J. Phenotype of Normal Hairline Maturation. Facial Plast Surg Clin North Am. 2013; 21(3):317-24.

17. Ordu KS., Agi CE. Inheritance pattern of hairline shape amongst Nigerian population. Int. J. Curr. Microbiolol. App. Sci. 2014; 3(2):61-5.

18. Ausrine Ramanauskaite and Ralph M Trüeb. Facial Papules in Fibrosing Alopecia in a Pattern Distribution (Cicatricial Pattern Hair Loss). Int J Trichology 2015 JulSep; 7(3) 119-122. 2015; 1(2):97-105.

19. Gupta AK., Foley KA., Lyons DCA. An algorithm for the diagnosis of female hair loss. J Am Acad Dermatol. 2015; 72 ( 5 ) : A B 111 . D i s p o n i b l e e n : http://linkinghub.elsevier.com/retrieve/pii/S0190962215 005770

20. Guyuron B, Gatherwright J, Totonchi A, Ahmadian R, Farajipour N. Cessation of hairline recession following open forehead rejuvenation. Plast Reconstr Surg [Internet]. 2014; 133(1):1e-6e. Disponible en: http://ovidsp.ovid.com/ovidweb.cgi?T=JS\&PAGE=refer ence \&D=medl \&NEWS=N\&AN=24374683

21. Serrano-Falcón C, Fernández-Pugnaire MA., SerranoOrtega S. Evaluación del pelo y cuero cabelludo: tricograma. Actas Dermosifiliogr. 2013; 104(10):867-76.

22. Samrao A., Chew A.L, Price V. Frontal fibrosing alopecia: A clinical review of 36 patients. $\mathrm{Br} \mathrm{J}$ Dermatol. 2010; 163(6):1296-300. 